\title{
A Nonrigid Image Registration Framework for Identification of Tissue Mechanical Parameters
}

\author{
Petr Jordan ${ }^{1,3}$, Simona Socrate ${ }^{2}$, Todd E. Zickler ${ }^{1}$, and Robert D. Howe ${ }^{1,3}$ \\ ${ }^{1}$ Harvard School of Engineering and Applied Sciences, Cambridge, MA \\ ${ }^{2}$ Department of Mechanical Engineering, MIT, Cambridge, MA \\ ${ }^{3}$ Harvard-MIT Division of Health Sciences and Technology, Cambridge, MA
}

\begin{abstract}
We present a modular framework for mechanically regularized nonrigid image registration of 3D ultrasound and for identification of tissue mechanical parameters. Mechanically regularized deformation fields are computed from sparsely estimated local displacements. We enforce image-based local motion estimates by applying concentrated forces at mesh nodes of a mechanical finite-element model. The concentrated forces are generated by the elongation of regularization springs connected to the mesh nodes as their free ends are displaced according to local motion estimates. The regularization energy corresponding to the potential energy stored in the springs is minimized when the mechanical response of the model matches the observed response of the organ. We demonstrate that this technique is suitable for identification of material parameters of a nonlinear viscoelastic liver model and demonstrate its benefits over traditional indentation methods in terms of improved volumetric agreement between the model response and the experiment.
\end{abstract}

\section{Introduction}

Mechanically accurate nonrigid registration of volumetric medical image data is an increasingly important aspect of guiding surgical procedures involving deformations of solid organs. Image-guided tasks, such as MRI tumor localization during brain shift, needle biopsy, prostate brachytherapy and others require a close interplay of computational biomechanical models with preoperative and intraoperative imaging. Considering the complexity of the mechanical response of solid organs, it is necessary to use properly formulated biomechanical organ models capable of capturing a nonlinear, rate-dependent, viscoelastic response under large deformation. Additionally, the significant subject-to-subject biological variability contributes to a strong motivation and clinical need for patientspecific (personalized) models, which may be generated and parameterized with clinically feasible testing protocols.

A wide array of sophisticated methods has been developed for mechanically constrained nonrigid registration of intraoperative brain [1] 2] and liver [4] deformations, cardiac motion [5] [6, breast deformation during mammography [7, as well as applications for prostate [8, and other organ systems. At the moment, the use of state-of-the-art biomechanical organ models in nonrigid image registration is hindered by the requirement of custom finite-element solvers,

D. Metaxas et al. (Eds.): MICCAI 2008, Part II, LNCS 5242, pp. 930 938, 2008.

(C) Springer-Verlag Berlin Heidelberg 2008 


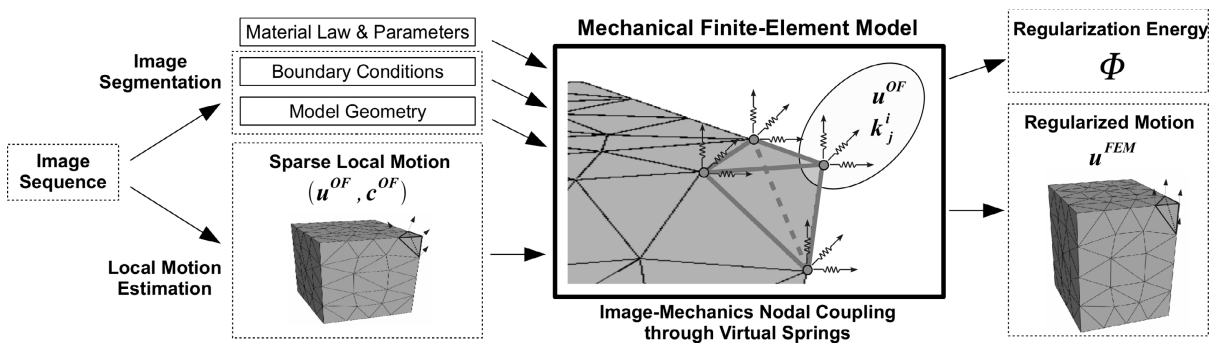

Fig. 1. In the proposed mechanical regularization framework, sparse local motion estimates are coupled to a mechanical finite-element model as lumped body forces applied by displaced regularization springs. This results in a mechanically constrained deformation field $\mathbf{u}^{F E M}$ and regularization energy $\Phi$.

mandated by the inherent coupling between the image-domain components of the algorithm and the biomechanical computational methods.

To address these limitations, we propose a modular registration framework, which relies on mechanical springs attached at nodal mesh locations to couple image-based motion estimates to a mechanical finite-element model. Such approach results in a separation of the image motion estimation scheme and offers the flexibility to use commercial finite-element solvers and complex constitutive material laws from the biomechanics community. Therefore, the most significant contribution of this method is in applications with complex constitutive laws.

\section{Methods}

Our general regularization framework (Fig. 1) links local image motion to a mechanical model to provide a global and mechanically accurate dense motion field. We propose to deform a mechanical model by applying concentrated forces at nodal locations. Three regularization springs attached to each node of the model apply lumped body forces in the 3 orthogonal coordinate directions. The freeend regularization springs displacements $\mathbf{u}^{O F}$ are obtained from a local motion estimate, which, in our implementation, is derived from the Lucas-Kanade optical flow method 9 . The spring stiffness is adjusted to reflect local nodal stiffness of the mechanical model and the motion estimate confidence $\mathbf{c}^{O F}$, which reflects the textural content in the neighborhood. The mechanically regularized field $\mathbf{u}^{F E M}$ is the displacement field obtained from the solution of the mechanically deformed finite-element model.

\subsection{Local Optical Flow Estimation}

In this modular architecture, any algorithm can be used to estimate the local optical flow $\mathbf{u}^{O F}$. As an example, we use a modified Lucas-Kanade algorithm. The traditional differential optical flow techniques, such as the Lucas-Kanade 
method, rely on two fundamental assumptions: frame-to-frame intensity constancy and local intensity gradient constancy. Under these assumptions the motion of each voxel is constrained by the optical flow equation

$$
\frac{\partial I}{\partial x} u_{x}+\frac{\partial I}{\partial y} u_{y}+\frac{\partial I}{\partial z} u_{z}+\frac{\partial I}{\partial t}=0
$$

where $I(x, y, z, t)$ is the voxel intensity and $\left\{u_{x}, u_{y}, u_{z}\right\}$ are vector components of the displacement. Since the optical flow constraint for a single voxel is illposed, the solution of the Lucas-Kanade algorithm relies on additional motion assumptions within the local neighborhood. We sample the neighborhood of each mesh node (all neighboring tetrahedra) and assemble a system of equations weighted by the corresponding elemental shape functions $N_{i}$ (see [10] for the linear tetrahedral shape function definition).

$$
N_{i} \frac{\partial I_{i}}{\partial x} u_{x}^{O F}+N_{i} \frac{\partial I_{i}}{\partial y} u_{y}^{O F}+N_{i} \frac{\partial I_{i}}{\partial z} u_{z}^{O F}=-N_{i} \frac{\partial I_{i}}{\partial t}
$$

Using the linear tetrahedral shape function as the nodal neighborhood weighting functions, the local system of optical flow equations can be rewritten as

$$
\mathbf{A} u^{O F}=\mathbf{b}, \quad \mathbf{A}_{\mathbf{i j}}=N_{i} \frac{\partial I_{i}}{\partial j}, \quad \mathbf{b}_{\mathbf{i}}=-N_{i} \frac{\partial I_{i}}{\partial t},
$$

where $i$ is the voxel index and $j=\{x, y, z\}$. The nodal displacement can be recovered as the least-squares solution to this linear system. Local motion $\mathbf{u}^{O F}$ is computed at each mesh node, providing a globally unconstrained set of local motion estimates. Each nodal motion estimate has an associated confidence $\mathbf{c}^{\mathrm{OF}}$. Traditionally, this confidence is computed from the three eigenvectors (direction of confidence) and eigenvalues (level of confidence) of the square $\mathbf{A}^{\mathbf{T}} \mathbf{A}$ matrix. However, to account for the variability of local neighborhood size throughout the mesh, we follow an alternative approach in which we compute the texturedependent confidence by summing the absolute values of image gradients in the nodal neighborhood, such that

$$
c_{j}^{O F}=\sum_{i=1}^{n} N_{i}\left|\frac{\partial I_{i}}{\partial j}\right|
$$

where $i=\{1, . ., n\}$ are all voxels contained in elements surrounding the node of interest and $j=\{x, y, z\}$. The value of $c_{j}^{O F}$ is subsequently normalized by the largest value contained in the image volume, such that $c_{j}^{O F} \in[0,1]$.

\subsection{Mechanically Regularized Deformation}

Once the local motion estimates and the associated confidence levels are computed, the mechanical model is deformed by the forces applied through regularization springs with one end attached to the nodes of the mechanical mesh, and one end constrained to match the displacement corresponding to local image 
motion. To provide a conceptual interpretation of this registration approach, the deformation of a simple one-dimensional continuum mechanics model (beam) is described in Fig. 2. We address two types of problems: the class of problems where boundary conditions are unknown (shown in Fig. 2, left) and well-posed boundary value problems with fully specified boundary conditions (Fig. 2, right).

The displacement field $\mathbf{u}^{F E M}$ is the equilibrium field computed by the finiteelement solver, minimizing the total potential energy of the system, which includes the strain energy stored in the continuum model and the potential energy in the regularization springs. Noisy $\mathbf{u}^{F E M}$ fields are penalized by the strain energy associated with the high local displacement gradients of the continuum model (beam) and excessively smoothed $\mathbf{u}^{F E M}$ fields are penalized by the increased potential energy of the regularization springs defined as

$$
U_{S}=\sum_{i=1}^{N} \sum_{j}\left(\frac{1}{2} \mathbf{k}_{j}^{i}\left(\mathbf{d}_{j}^{i}\right)^{2}\right),
$$

where $j=\{x, y, z\}, N$ is the number of attached regularization springs, $\mathbf{k}_{j}^{i}$ is the spring stiffness, and the spring distension $\mathbf{d}_{j}^{i}$ is defined as $\mathbf{d}_{j}^{i}=\mathbf{u}^{O F}-\mathbf{u}^{F E M}$. In order to relate the image-based confidence values to physically relevant springs stiffnesses, each stiffness is obtained not only as a function of local image texture, but also of the local nodal stiffness of the mechanical model. Therefore, the stiffness of each regularization spring is computed as

$$
\mathbf{k}_{j}^{i}=\beta \mathbf{K}_{j}^{i} \mathbf{c}_{i j}^{O F},
$$

Unknown Boundary Conditions

\section{Reference configuration}

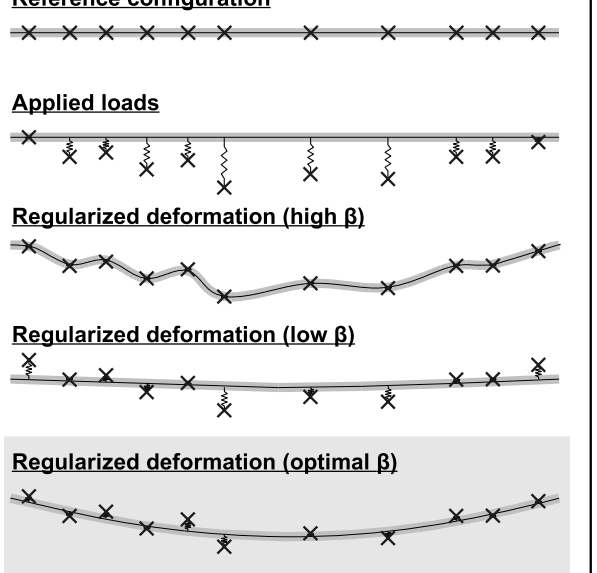

Fully Specified Boundary Conditions

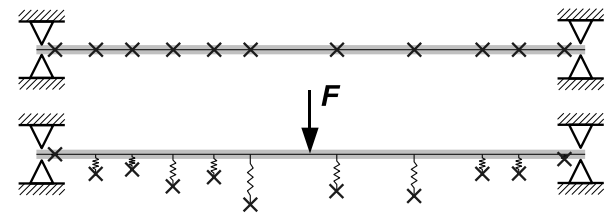

Regularized deformation $(\beta \approx 1)$
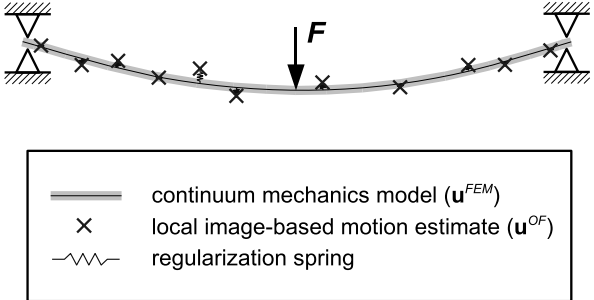

Fig. 2. Deformation of a continuum mechanics model (beam) with image-based forces in the form of elongated regularization springs. The class of problems where boundary conditions are unknown is shown on the left. A well-posed boundary value problem with fully specified boundary conditions is shown on the right. 
where $i$ is the node index, $\beta$ is the regularization coefficient, and $\mathbf{K}_{j}^{i}$ is the nodal stiffness of the mechanical model. Nodal stiffness values are the diagonal members of the global stiffness matrix, assembled from contributions of elemental stiffness matrices (see [10] for details). The time-evolving nodal stiffness is computed by the finite-element solver and is updated at every solution increment.

The balance between image-based and mechanics-based contributions of the final regularized displacement is governed by the stiffness of the attached regularization springs. As shown in Eq. 6, the spring stiffness contains a scaling parameter $\beta$. A judicious choice of the parameter $\beta$ ensures that an optimal balance between the continuum body strain energy and spring potential energy costs is achieved. For problems in which the optical field data is the only available information, the parameter $\beta$ must be sufficiently high to impose the deformation on the body. Conceptually, for the schematic in Fig. 2, if the deformation of the beam is only driven by the displacement of the free ends of the springs, excessive compliance of the springs will result in underestimation of the deformation. For well-posed boundary value problems (BVPs), in which either traction or displacements are known over the entire boundary, the deformation of the continuum (finite-element) model could be driven entirely by these boundary conditions. We consider two applications of the proposed framework, where 3DUS imaging can be combined with surface (boundary) information to provide (1) accurate reconstruction of organ inner field deformation and (2) enhanced measurement of the constitutive response of an organ. For inner field reconstruction, the boundary conditions drive the global deformation, while the regularization springs impose local constraints. The spring stiffness does not need to exceed the model stiffness, therefore values of $\beta \approx 1$ are more appropriate. When measuring the constitutive response of an organ, the normalized potential energy in the springs, defined as

$$
\Phi\left(\mathbf{p}_{n}\right)=\sum_{i=1}^{N} \sum_{j}\left(\frac{\frac{1}{2} \mathbf{k}_{j}^{i}\left(\mathbf{d}_{j}^{i}\right)^{2}}{\frac{1}{2} \beta \mathbf{K}_{j}^{i}}\right)=\sum_{i=1}^{N} \sum_{j} \mathbf{c}_{i j}^{O F}\left(\mathbf{u}_{i j}^{O F}-\mathbf{u}_{i j}^{F E M}\right)^{2}
$$

is a measure of model-experiment agreement. The energy is normalized by model stiffness to prevent artificial bias towards compliant models. Imperfect models are associated with higher levels of regularization energy. In principle, if the model were perfect, the regularization energy would be a measure only of the noise in the optical flow. The magnitude of the regularization energy, therefore, can be considered a measure of the accuracy of a constitutive formulation, and minimization algorithms can be used for optimal tissue parameter selection.

\subsection{Porcine Liver Parameter Identification}

In this tissue parameter estimation study, we demonstrate that material parameters obtained from traditional indentation studies may not be unique even for relatively simple material constitutive laws, as numerous parameter combinations may be selected to obtain a good force-displacement agreement between the model response and the experiment. A perfused porcine liver was indented 
(0.2 mm/s load-unload cycle, $10 \mathrm{~mm}$ total displacement) with a cylindrical indenter (12mm diameter) actuated by Bose Electroforce ELF 3200 material testing system (Bose Corporation, Eden Prairie, MN, USA). The volumetric deformation of the liver was acquired with 3D ultrasound probe (SONOS 7500, Philips Medical Systems, Andover, MA, USA) placed below the tissue sample. The volumetric image data contains relatively slow deformations (maximum tissue displacement is less than 0.3 voxels per frame) and the liver parenchyma produces rich textural content under 3DUS (see Figure 3). In our related work, we demonstrate that our method has good tissue tracking accuracy against manually tracked tissue landmarks in ex vivo liver indentation and present a quantitative error analysis using synthetic deformation sequences [11.

The 3DUS volume was registered to finite-element model (Abaqus 6.7, SIMULIA, Providence, RI, USA) with simplified cylindrical geometry (10 cm diameter, $3 \mathrm{~cm}$ height). The geometry, the boundary conditions (force $F(t)$ applied at the tip of the indenter, and constrained displacement at the bottom surface of the organ), and the constitutive law were incorporated into the regularizer, while initializing the material parameters with an appropriate initial estimate. We selected a relatively simple nonlinear viscoelastic constitutive relationship in the form of a $2^{\text {nd }}$-order reduced polynomial hyperelastic formulation for the instantaneous response and a $1^{\text {st }}$-order Prony series relaxation of the shear modulus [12. The hyperelastic strain energy of this constitutive law is defined as

$$
U=C_{1}\left(I_{1}-3\right)+C_{2}\left(I_{1}-3\right)^{2}+\frac{1}{D_{1}}\left(J_{e l}-1\right)^{2}
$$

where $C_{1}, C_{2}$, and $D_{1}$ are the material parameters, $I_{1}$ is the $1^{\text {st }}$ stretch invariant, and $J_{e l}$ is the elastic volumetric stretch. The relaxation of the shear modulus $G(t)$ is captured by a $1^{\text {st }}$-order Prony series

$$
G(t)=G_{0}\left(g_{\infty}+g_{1} e^{-t / \tau_{1}}\right)
$$

where $G_{0}$ is the instantaneous shear strain modulus (computed from Eq. 8), $G_{0} g_{\infty}$ is the equilibrium shear strain modulus, $g_{1}=1-g_{\infty}$ is the relative amplitude of the relaxation, and $\tau_{1}$ is the relaxation time constant.

We used a nonlinear optimization scheme, a bounded downhill simplex method, to iteratively evolve the material parameters $\left(C_{1}, C_{2}, D_{1}, g_{1}, \tau_{1}\right)$ and minimize the rate form of the regularization energy $\Phi\left(\mathbf{p}_{n}\right)$.

\section{Results}

Traditionally, tissues are characterized based on time-displacement-force relationships obtained in a specific mode of deformation (tension, compression, shear, indentation, etc.). In this study we present 3 model parameter sets, which exhibit nearly identical indentation force-displacement response (Fig. 4, left). Their volumetric response, however, is significantly different, and our method offers means for differentiating between these models. The parameter set \#3 was obtained by 


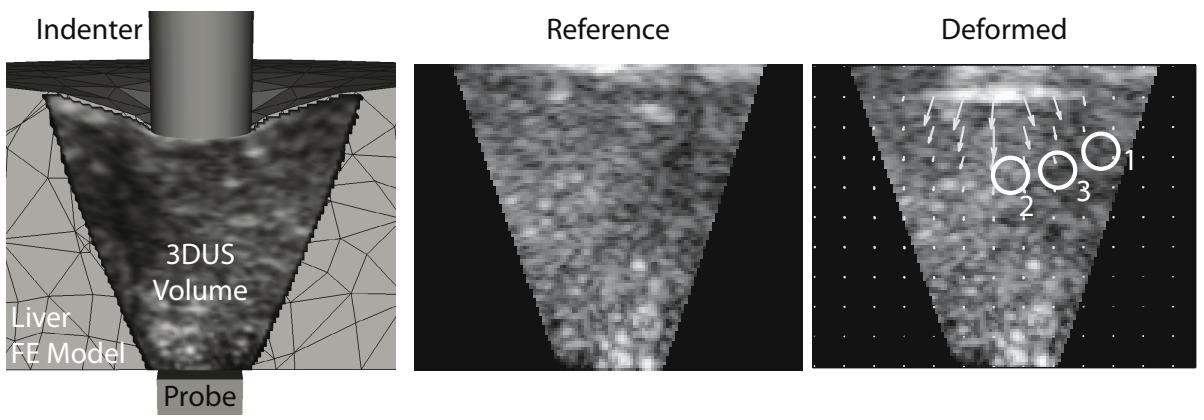

Fig. 3. Left: A cut through the deforming finite-element model and 3DUS sequence. Middle: Slice through the undeformed 3DUS volume. Right: Slice through the deforming volume $(\mathrm{t}=1.2 \mathrm{~s})$, showing the local optical flow and the 3 locations evaluated.
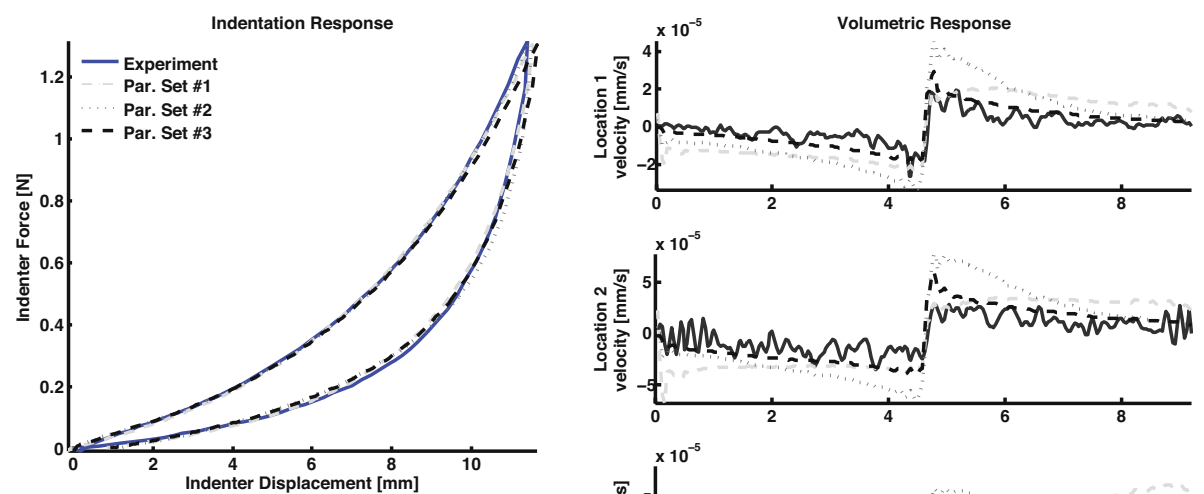

\begin{tabular}{l|r|r|c|c|c} 
& \multicolumn{1}{|c|}{$C_{1}$} & \multicolumn{1}{c|}{$C_{2}$} & $D_{1}$ & $g_{1}$ & $\tau_{1}$ \\
\hline Par. Set \#1 & 4.0 & 47.8 & $1.0 \mathrm{E}-4$ & 0.890 & 0.223 \\
Par. Set \#2 & 181.5 & 631.8 & $3.0 \mathrm{E}-3$ & 0.788 & 0.159 \\
Par. Set \#3 & 88.8 & 238.0 & $4.5 \mathrm{E}-4$ & 0.736 & 0.259
\end{tabular}

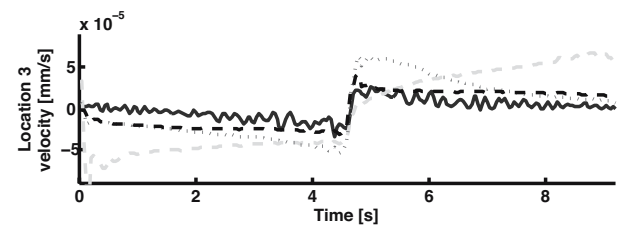

Fig. 4. While the indentation response is virtually identical (left) for the 3 parameter sets chosen, the volumetric material deformation provides unique tissue response signature. The vertical component of mesh node velocity is shown on the right.

fitting all parameters of the model, maximizing the volumetric model-experiment agreement (material velocities at 3 selected locations shown in Fig. 4, right). In contrast, the parameter sets \#1 and \#2 were identified without image data by fitting the force-displacement indentation response only. Since the volumetric compliance $D_{1}$ cannot be observed directly in conventional indentation, $D_{1}$ was assumed to be comparatively low and high, with respect to parameter set \#3. When image data is considered, minimizations seeded from parameter sets \#1 and \#2 both converge to parameter set \#3, suggesting that the volumetric parameter identification method may remove degeneracies in the parameter space and may offer an objective function with a unique minimum. 


\section{Discussion}

We have presented a nonrigid registration algorithm regularized by a mechanical finite-element model suitable for applications in 3D ultrasound tissue tracking and material parameter estimation. One of the key contribution of this method is the image-mechanics coupling approach, which uses regularization springs attached at nodal locations to enforce image-based motion estimates. This approach avoids the need for direct computation of image forces and provides an intuitive assignment of image-based motion confidence, reflecting the spatial variations in texture quality. Additionally, a key feature of this approach is the modular nature of its formulation, under which the choices of image similarity measure, local search algorithm, image-mechanics confidence mapping, and most importantly, the mechanical model's material law, are completely independent. The implementation of this method is not significantly more complex than similar methods, as it leverages the power of commercially available finiteelement solvers. This is of high importance, as it enables the use of nonlinear, viscoelastic material models of arbitrary complexity without the need for a reliable custom-made FEM solver. This flexibility becomes increasingly important with the ongoing progress and increasing complexity of constitutive mechanical models formulated for large-strain behavior of soft tissues.

The proposed registration framework is suitable for applications in mechanical parameter identification and provides good accuracy and sensitivity to the bulk and shear components of the material response. This ability is of high importance to future characterization of complex constitutive laws and is appealing for in vivo applications, where organ boundaries cannot be directly controlled.

Acknowledgements. This work was supported by the US Army (DAMD 1701-1-0677 and DAAD-19-02-D0002), the NIH (R01 HL073647-01), the Joint Improvised Explosive Devices Defeat Organization (W911NF-07-1-0035), and the Harvard-MIT Division of Health Sciences and Technology.

\section{References}

1. Ferrant, M., et al.: Registration of 3-D Intraop. MR Images of the Brain using a Finite-Element Biomech. Model. IEEE T Med. Imaging 20(12), 1384-1397 (2001)

2. Skrinjar, O., et al.: Model-driven Brain Shift Compensation. Med. Image Anal. 6(4), 361-373 (2002)

3. Clatz, O., et al.: Robust Nonrigid Registration to Capture Brain Shift from Intraoperative MRI. IEEE T Med. Imaging 24(11), 1417-1427 (2005)

4. Cash, D.M., et al.: Concepts and Preliminary Data Toward the Realization of Image-guided Liver Surgery. J. Gastrointest Surg. 11(7), 844-859 (2007)

5. Papademetris, X., et al.: Estimation of 3-D Left Ventricular Deformation from Med. Images using Biomech. Models. IEEE T Med. Imaging 21(7), 786-800 (2002)

6. Sermesant, M., et al.: Deformable Biomechanical Models: Application to 4D Cardiac Image Analysis. Med. Image Anal. 7(4), 475-488 (2003) 
7. Roose, L., et al.: Biomechanically Based Elastic Breast Registration using Mass Tensor Simulation. In: Larsen, R., Nielsen, M., Sporring, J. (eds.) MICCAI 2006. LNCS, vol. 4191, pp. 718-725. Springer, Heidelberg (2006)

8. Alterovitz, R., et al.: Registration of MR Prostate Images with Biomechanical Modeling and Nonlinear Parameter Estimation. Med. Phys. 33(2), 446-454 (2006)

9. Lucas, B.D., Kanade, T.: An Iterative Image Registration Technique with an Application to Stereo Vision. In: Proc. IJCAI, pp. 674-679 (1981)

10. Zienkiewicz, O.C.: The Finite Element Method. McGraw-Hill, New York (1977)

11. Jordan, P.: Image-Based Mechanical Characterization of Soft Tissue using Three Dimensional Ultrasound. Ph.D. Thesis, Harvard University (2008)

12. SIMULIA, Providence, RI: ABAQUS Theory Manual (2007) 